\title{
Quasi-StaticTransient Thermal Stresses in a Dirichlet's thin Solid Cylinder with Internal Moving Heat Source
}

\author{
${ }^{1}$ D. T. Solanke, ${ }^{2}$ M. H. Durge \\ ${ }^{I}$ Sudhakar Naik and UmashankarKhetancollege Akola, Maharashtra State, India. \\ ${ }^{2}$ Anand NiketanCollge, Warora, Maharashtra State, India.
}

\begin{abstract}
This paper concern with transient non-homogeneousthermoelastic problem with Dirichlet's boundary condition inthin solid cylinder of isotropic material of radius $a$, and height $h$, occupying the space $0 \leq r \leq a$, $-\frac{h}{2} \leq z \leq \frac{h}{2}, 0 \leq \phi \leq 2 \pi$ having initial temperature $f(r, \phi, z)$ placed in an ambient temperature zero. The cylinder is subjected to the activity of moving heat source along circular trajectory of radius $r_{0}$, where $0<r_{0}<a$, around the centre of the cylinder with constant angular velocity $\omega$. Heat conduction equation containing heat generation term is solved by applying integral transform technique and Green's theorem is adopted in deducing the solution of heat conduction equation. The solution is obtained in a series form of Bessel function and trigonometric function and derived thermal stresses.
\end{abstract}

Keywards: Dirichlet's solid thin cylinder, moving heat source, thermal stresses, Green's theorem.

\section{Introduction:}

During the second half of $20^{\text {th }}$ century, non-isothermal problems of the theoryof elasticity became increasingly important. This is due to their wide application in diverse fields. The high velocities of modern aircraft give rise to aerodynamic heating, which produces intense thermal stresses that reduce the strength of aircraft structure.

In thispresent paper we determine temperature, thermal stresses, in a Dirichlet's thin solid cylinder, determined by $0 \leq r \leq a,-\frac{h}{2} \leq z \leq \frac{h}{2}, 0 \leq \phi \leq 2 \pi$ with internal moving heat source. Heat conduction equation with heat generation term is solved by applying integral transform technique and Green's theorem. Solution is obtained in series form of Bessel function and trigonometric function. The direct problems is very important in view of its relevance to various industrial mechanics subjected to heating such as main shaft of lathe, turbines and the role of rolling millfor base of furnace boiler of thermal power plant, gas power plant and measurement of aerodynamic heating.

\section{Formulation of the Heat conduction problem:}

Consider a thin solid cylinder of isotropic material of radius $a$ and thickness $h$ occupying the space $0 \leq r \leq a,-\frac{h}{2} \leq z \leq \frac{h}{2}, 0 \leq \phi \leq 2 \pi$ having initial temperature $f(r, \phi, z)$ placed in an ambient temperature zero. The cylinder is subjected to the activity of moving heat source which changes its place along circular trajectory of radius $r_{0}$, where $0<r_{0}<a$, around the centre of the cylinder with constant angular velocity $\omega$. The activity of moving heat source and initial temperature of the cylinder may cause the generation of heat due to nuclear interaction that may be a function of position and time in the form $g(r, \phi, z, t) \mathrm{w} / \mathrm{s}^{3}$. The temperature distribution of the solid cylinder is described by the differential equation of heat conduction with heat generation term as in [2] page no. 232 is given by

$\nabla^{2} T+\frac{1}{k} g=\frac{1}{\alpha} \frac{\partial T}{\partial t}$

Where $T=T(r, \phi, z)$ is temperature distribution, $k$ is thermal conductivity of the material of the cylinder, $\alpha=\frac{k}{\rho C_{p}}$ is thermal diffusivity, $\rho$ is density , $C_{p}$ is specific heat of the material and $g$ is the volumetric energy generation term in the cylinder. Where $\nabla^{2}$ is Laplacian operator in cylindrical coordinates and $\nabla^{2}=\frac{\partial^{2}}{\partial r^{2}}+\frac{1}{r} \frac{\partial}{\partial r}+\frac{1}{r^{2}} \frac{\partial^{2}}{\partial \phi^{2}}+\frac{\partial^{2}}{\partial z^{2}}$ 
Now consider an instantaneously moving heat source $g_{s}{ }^{i}$ located at a point $\left(r_{0}, \phi^{\prime}, \xi\right)$ and releasing its energy spontaneously at time $\tau$. Such volumetric heat source in cylindrical coordinates is given by

$g(r, \phi, z, t)=g_{s}{ }^{i} \frac{1}{2 \pi r} \delta\left(r-r_{0}\right) \delta\left(\phi-\phi^{\prime}\right) \delta(z-\xi) \delta(t-\tau)$

Hence above equation reduces to

$\nabla^{2} T+g_{s}^{i} \frac{1}{2 \pi k r} \delta\left(r-r_{0}\right) \delta\left(\phi-\phi^{\prime}\right) \delta(z-\xi) \delta(t-\tau)=\frac{1}{\alpha} \frac{\partial T}{\partial t}$

$\phi^{\prime}=\omega t$

With initial and homogeneous boundary conditions,

$T=0 \quad$ at $\quad r=a$

$T=0 \quad$ at $\quad z=-\frac{h}{2}$

$T=0 \quad$ at $\quad z=\frac{h}{2}$

$T=f(r, \phi, z)$ at $t=0$

\section{Formulation of thermoelastic Problem:}

Let us introduce a thermal stress function $\chi$ related to component of stress in the cylindrical coordinates system as in [3]

$$
\begin{aligned}
& \sigma_{r r}=\frac{1}{r} \frac{\partial \chi}{\partial r}+\frac{1}{r^{2}} \frac{\partial^{2} \chi}{\partial r^{2}} \\
& \sigma_{\phi \phi}=\frac{\partial^{2} \chi}{\partial r^{2}} \\
& \sigma_{r \phi}=-\frac{\partial}{\partial r}\left(\frac{1}{r} \frac{\partial \chi}{\partial \phi}\right)
\end{aligned}
$$

The boundary condition for a traction free body are

$\sigma_{r r}=0, \sigma_{r \phi}=0$ at $r=a$

Where $\chi=\chi_{c}+\chi_{p}$

Where $\chi_{c}$ is complementary solution and $\chi_{p}$ is particular solution and

$\chi_{c}$ satisfies the equation $\nabla^{4} \chi_{c}=0$

$\chi_{p}$ satisfies the equation $\nabla^{4} \chi_{p}=-\lambda E \nabla^{2} \Gamma$

Where $\Gamma$ is temperature change $\Gamma=T-T_{i}, \quad T_{i}$ is initial temperature

$\nabla^{2}=\frac{\partial^{2}}{\partial r^{2}}+\frac{1}{r} \frac{\partial}{\partial r}+\frac{1}{r^{2}} \frac{\partial^{2}}{\partial \phi^{2}}$ since cylinder is thin, $\mathrm{z}$ component is negligible

\section{Solution of the Heat conduction problem:}

We define integral transform of $T(r, \phi, z)$ by

$\bar{T}=\int_{-\frac{h}{2}}^{\frac{h}{2}} \int_{0}^{2 \pi} \int_{0}^{a} T(r, \phi, z) r J_{v}\left(\beta_{m} r\right) \cos v\left(\phi-\phi^{\prime}\right) \sin \alpha_{n}\left(z+\frac{h}{2}\right) d r d \phi d z$

And its inverse by

$T=\frac{4}{\pi h a^{2}} \sum_{m=0}^{\infty} \sum_{n=0}^{\infty} \sum_{v=0}^{\infty} \bar{T} \frac{J v\left(\beta_{m} r\right) \cos v\left(\phi-\phi^{\prime}\right) \sin \alpha_{n}\left(z+\frac{h}{2}\right)}{\left[J_{v+1}\left(\beta_{m} a\right)\right]^{2}}$

Where $v=0,1,2,3 \ldots \ldots$.

$\beta_{m}$ Is root of the transcendental equation $J_{v}\left(\beta_{m} a\right)=0$ 
$\alpha_{n}=\frac{p \pi}{h}, \quad p=0,1,2,3 \ldots$.

By taking integral transform of equation (3.1) and using following Green's theorem

$\int_{R} \nabla^{2} T \psi_{k} d v=\int_{R} T \nabla^{2} \psi_{k} d v+\sum_{i=1}^{N} \int_{s_{i}}\left[\psi_{k} \frac{\partial T}{\partial n_{i}}-T \frac{\partial \psi_{k}}{\partial n_{i}}\right] d s_{i}$

Which yield as

$\frac{d \bar{T}}{d t}+\alpha\left(\beta_{m}{ }^{2}+\alpha_{n}{ }^{2}\right) \bar{T}=g_{s}{ }^{i} \frac{\alpha}{2 \pi k} J_{v}\left(\beta_{m} r_{0}\right) \sin \alpha_{n}\left(\xi+\frac{h}{2}\right) \delta(t-\tau)$

This is linear differential equation of first order. By solving this we arrive at

$\bar{T}=\left[\bar{f}\left(\beta_{m}, v, \alpha_{n}\right)+\frac{\alpha}{2 \pi k} g_{s}{ }^{i} J_{v}\left(\beta_{m} r_{0}\right) \sin \alpha_{n}\left(\xi+\frac{h}{2}\right) e^{\alpha\left(\beta_{m}{ }^{2}+\alpha_{n}{ }^{2}\right) \tau}\right] e^{-\alpha\left(\beta_{m}{ }^{2}+\alpha_{n}{ }^{2}\right) t}$

Where $\quad \bar{f}=\int_{-\frac{h}{2}}^{\frac{h}{2}} \int_{0}^{2 \pi} \int_{0}^{a} f(r, \phi, z) r J_{v}\left(\beta_{m} r\right) \cos v\left(\phi-\phi^{\prime}\right) \sin \alpha_{n}\left(z+\frac{h}{2}\right) d r d \phi d z$

Taking inverse integral transform we finally arrive at

$$
\begin{gathered}
T=\frac{4}{\pi h a^{2}} \sum_{m, n, v=0}^{\infty} \frac{J_{v}\left(\beta_{m} r\right) \cos v\left(\phi-\phi^{\prime}\right) \sin \alpha_{n}\left(z+\frac{h}{2}\right)}{\left[J_{v+1}\left(\beta_{m} a\right)\right]^{2}}\left[\bar{f}\left(\beta_{m}, v, \alpha_{n}\right)+\frac{\alpha}{2 \pi k} g_{s}{ }^{i} J_{v}\left(\beta_{m} r_{0}\right) \sin \alpha_{n}\left(\xi+\frac{h}{2}\right) e^{\alpha\left(\beta_{m}{ }^{2}+\alpha_{n}{ }^{2}\right) \tau}\right] e^{-\alpha\left(\beta_{m}{ }^{2}+\alpha_{n}{ }^{2}\right) t} \\
\Gamma=\frac{4}{\pi h a^{2}} \sum_{m, n, v=0}^{\infty} \frac{J_{v}\left(\beta_{m} r\right) \cos v\left(\phi-\phi^{\prime}\right) \sin \alpha_{n}\left(z+\frac{h}{2}\right)}{\left[J_{v+1}\left(\beta_{m} a\right)\right]^{2}}\left[\bar{f}\left(\beta_{m}, v, \alpha_{n}\right)+\frac{\alpha}{2 \pi k} g_{s}{ }^{i} J_{v}\left(\beta_{m} r_{0}\right) \sin \alpha_{n}\left(\xi+\frac{h}{2}\right) e^{\alpha\left(\beta_{m}{ }^{2}+\alpha_{n}{ }^{2}\right) \tau}\right] \\
\\
{\left[e^{-\alpha\left(\beta_{m}{ }^{2}+\alpha_{n}{ }^{2}\right) t}-1\right]}
\end{gathered}
$$

\section{Solution of Thermoelastic Problem:}

Let suitable form of $\chi_{c}$ satisfying equation (4.6) be

$$
\chi_{c}=\sum_{v=0}^{\infty}\left(A r^{v+2}+B r^{-v+2}\right) \cos v\left(\phi-\phi^{\prime}\right)+\left(C r^{v+2}+D r^{-v+2}\right) \sin v\left(\phi-\phi^{\prime}\right)
$$

Let suitable form of $\chi_{p}$ satisfying equation (4.7) be

$$
\begin{gathered}
\chi_{p}=\sum_{m, n, v=0}^{\infty} \frac{\lambda E}{\beta_{m}^{2}} J_{v}\left(\beta_{m} r\right) \cos v\left(\phi-\phi^{\prime}\right) \sin \alpha_{n}\left(z+\frac{h}{2}\right)\left[\bar{f}\left(\beta_{m}, v, \alpha_{n}\right)+\frac{\alpha}{2 \pi k} g_{s}^{i} J_{v}\left(\beta_{m} r_{0}\right) \sin \alpha_{n}\left(\xi+\frac{h}{2}\right) e^{\alpha\left(\beta_{m}{ }^{2}+\alpha_{n}{ }^{2}\right) \tau}\right] \\
{\left[e^{-\alpha\left(\beta_{m}{ }^{2}+\alpha_{n}{ }^{2}\right) t}-1\right]}
\end{gathered}
$$

$\chi=\chi_{c}+\chi_{p}$ gives

$$
\chi=\sum_{v=0}^{\infty}\left(A r^{v+2}+B r^{-v+2}\right) \cos v\left(\phi-\phi^{\prime}\right)+\left(C r^{v+2}+D r^{-v+2}\right) \sin \left(\phi-\phi^{\prime}\right)+
$$

$$
\begin{gathered}
\sum_{m, n, v=0}^{\infty} \frac{\lambda E}{\beta_{m}{ }^{2}} J_{v}\left(\beta_{m} r\right) \cos v\left(\phi-\phi^{\prime}\right) \sin \alpha_{n}\left(z+\frac{h}{2}\right)\left[\bar{f}\left(\beta_{m}, v, \alpha_{n}\right)+\frac{\alpha}{2 \pi k} g_{s}{ }^{i} J_{v}\left(\beta_{m} r_{0}\right) \sin \alpha_{n}\left(\xi+\frac{h}{2}\right) e^{\alpha\left(\beta_{m}{ }^{2}+\alpha_{n}{ }^{2}\right) \tau}\right] \\
{\left[e^{-\alpha\left(\beta_{m}{ }^{2}+\alpha_{n}{ }^{2}\right) t}-1\right]}
\end{gathered}
$$

From (4.1) and (6.3) we obtain

$\sigma_{r r}=\sum_{v=0}^{\infty}\left\{\left[A\left(2+v-v^{2}\right) r^{v}+B\left(2-v-v^{2}\right) r^{-v}\right] \cos v\left(\phi-\phi^{\prime}\right)+\left[C\left(2+v-v^{2}\right) r^{v}+D\left(2-v-v^{2}\right) r^{-v}\right] \sin \left(\phi-\phi^{\prime}\right)\right\}+$ 


$$
\begin{aligned}
& \sum_{m, n, v=0}^{\infty} \frac{\lambda E}{\beta_{m}{ }^{2} r^{2}}\left[\beta_{m} r J_{v-1}\left(\beta_{m} r\right)-\left(v+v^{2}\right) J_{v}\left(\beta_{m} r\right)\right] \cos v\left(\phi-\phi^{\prime}\right) \sin \alpha_{n}\left(z+\frac{h}{2}\right) . \\
& {\left[\bar{f}\left(\beta_{m}, v, \alpha_{n}\right)+\frac{\alpha}{2 \pi k} g_{s}{ }^{i} J_{v}\left(\beta_{m} r_{0}\right) \sin \alpha_{n}\left(\xi+\frac{h}{2}\right) e^{\alpha\left(\beta_{m}{ }^{2}+\alpha_{n}{ }^{2}\right) \tau}\right]\left[e^{-\alpha\left(\beta_{m}{ }^{2}+\alpha_{n}{ }^{2}\right) t}-1\right] } \\
& \sigma_{\phi \phi}=\{ {\left.\left[A\left(v^{2}+3 v+2\right) r^{v}+B\left(v^{2}-3 v+2\right) r^{-v}\right] \cos v\left(\phi-\phi^{\prime}\right)+\left[C\left(v^{2}+3 v+2\right) r^{v}+D\left(v^{2}-3 v+2\right) r^{-v}\right] \sin v\left(\phi-\phi^{\prime}\right)\right\}+ } \\
& \sum_{m, n, v=0}^{\infty} \frac{\lambda E}{\beta_{m}{ }^{2}}\left[\frac{\beta_{m}}{r} J_{v+1}\left(\beta_{m} r\right)-\beta_{m}{ }^{2} J_{v}\left(\beta_{m} r\right)\right] \cos v\left(\phi-\phi^{\prime}\right) \sin \alpha_{n}\left(z+\frac{h}{2}\right) . \\
& {\left[\bar{f}\left(\beta_{m}, v, \alpha_{n}\right)+\frac{\alpha}{2 \pi k} g_{s}{ }^{i} J_{v}\left(\beta_{m} r_{0}\right) \sin \alpha_{n}\left(\xi+\frac{h}{2}\right) e^{\alpha\left(\beta_{m}{ }^{2}+\alpha_{n}{ }^{2}\right) \tau}\right]\left[e^{-\alpha\left(\beta_{m}{ }^{2}+\alpha_{n}{ }^{2}\right) t}-1\right] } \\
& \sigma_{r \phi}=\sum_{v=0}^{\infty}\left\{\left[A\left(v^{2}+v\right) r^{v}+B\left(-v^{2}+v\right) r^{-v}\right] \sin v\left(\phi-\phi^{\prime}\right)+\left[C\left(-v^{2}-v\right) r^{v}+D\left(v^{2}-v\right) r^{-v}\right] \cos v\left(\phi-\phi^{\prime}\right)\right\}+ \\
& \sum_{m, n, v=0}^{\infty} \frac{\lambda E v}{\beta_{m}{ }^{2} r^{2}}\left[\beta_{m} r J_{v-1}\left(\beta_{m} r\right)-(1+v) J_{v}\left(\beta_{m} r\right)\right] \sin v\left(\phi-\phi^{\prime}\right) \sin \alpha_{n}\left(z+\frac{h}{2}\right) . \\
& {\left[\bar{f}\left(\beta_{m}, v, \alpha_{n}\right)+\frac{\alpha}{2 \pi k} g_{s}{ }^{i} J_{v}\left(\beta_{m} r_{0}\right) \sin \alpha_{n}\left(\xi+\frac{h}{2}\right) e^{\alpha\left(\beta_{m}{ }^{2}+\alpha_{n}{ }^{2}\right) \tau}\right]\left[e^{-\alpha\left(\beta_{m}{ }^{2}+\alpha_{n}{ }^{2}\right) t}-1\right] }
\end{aligned}
$$

Applying condition (4.4) to (6.4) and (6.6) we obtain

$$
\begin{array}{r}
A=-\sum_{m, n, v=0}^{\infty} \frac{a^{-v} \lambda E}{2 v \beta_{m} a} J_{v-1}\left(\beta_{m} a\right) \sin \alpha n\left(z+\frac{h}{2}\right) \cdot\left[\bar{f}\left(\beta_{m}, v, \alpha_{n}\right)+\frac{\alpha}{2 \pi k} g_{s}^{i} J_{v}\left(\beta_{m} r_{0}\right) \sin \alpha_{n}\left(\xi+\frac{h}{2}\right) e^{\alpha\left(\beta_{m}{ }^{2}+\alpha_{n}{ }^{2}\right) \tau}\right] \\
{\left[e^{-\alpha\left(\beta_{m}{ }^{2}+\alpha_{n}{ }^{2}\right) t}-1\right]}
\end{array}
$$$$
B=\sum_{m, n, v=0}^{\infty} \frac{a^{v} \lambda E}{2 v \beta_{m} a} J_{v-1}\left(\beta_{m} a\right) \sin \alpha_{n}\left(z+\frac{h}{2}\right) \cdot\left[\bar{f}\left(\beta_{m}, v, \alpha_{n}\right)+\frac{\alpha}{2 \pi k} g_{s}{ }^{i} J_{v}\left(\beta_{m} r_{0}\right) \sin \alpha_{n}\left(\xi+\frac{h}{2}\right) e^{\alpha\left(\beta_{m}{ }^{2}+\alpha_{n}{ }^{2}\right) \tau}\right]
$$$$
\left[e^{-\alpha\left(\beta_{m}{ }^{2}+\alpha_{n}^{2}\right) t}-1\right]
$$

$C=0, D=0$

Substituting (6.7), (6.8) and (6.9) in above equations which finally reduces to

$$
\begin{aligned}
\sigma_{r r}= & \sum_{m, n, v=0}^{\infty}\left\{\left[a^{v}\left(2-v-v^{2}\right) r^{-v}-a^{-v}\left(2+v-v^{2}\right) r^{v}\right] \frac{J_{v-1}\left(\beta_{m} a\right)}{2 v \beta_{m} a}+\left[\frac{\beta_{m} r J_{v-1}\left(\beta_{m} r\right)-\left(v+v^{2}\right) J_{v}\left(\beta_{m} r\right)}{\beta_{m}{ }^{2} r^{2}}\right]\right\} . \\
& \lambda E \cos v\left(\phi-\phi^{\prime}\right) \sin \alpha_{n}\left(z+\frac{h}{2}\right) \cdot\left[\bar{f}\left(\beta_{m}, v, \alpha_{n}\right)+\frac{\alpha}{2 \pi k} g_{s}{ }^{i} J_{v}\left(\beta_{m} r_{0}\right) \sin \alpha_{n}\left(\xi+\frac{h}{2}\right) e^{\alpha\left(\beta_{m}{ }^{2}+\alpha_{n}{ }^{2}\right) \tau}\right] \\
\sigma_{\phi \phi}= & \sum_{m, n, v=0}^{\infty}\left\{\left[a^{v}\left(v^{2}-3 v+2\right) r^{-v}-a^{-v}\left(v^{2}+3 v+2\right) r^{v}\right] \frac{J_{v-1}\left(\beta_{m} a\right)}{2 v \beta_{m} a}+\left[\frac{\beta_{m} J_{v+1}\left(\beta_{m} r\right)-r \beta_{m}{ }^{2} J_{v}\left(\beta_{m} r\right)}{\left.r \beta_{m}{ }^{2}\right) t}\right]\right\} .
\end{aligned}
$$$$
\lambda E \cos v\left(\phi-\phi^{\prime}\right) \sin \alpha_{n}\left(z+\frac{h}{2}\right) .\left[\bar{f}\left(\beta_{m}, v, \alpha_{n}\right)+\frac{\alpha}{2 \pi k} g_{s}{ }^{i} J_{v}\left(\beta_{m} r_{0}\right) \sin \alpha_{n}\left(\xi+\frac{h}{2}\right) e^{\alpha\left(\beta_{m}{ }^{2}+\alpha_{n}{ }^{2}\right) \tau}\right]
$$ 


$$
\begin{gathered}
{\left[e^{-\alpha\left(\beta_{m}{ }^{2}+\alpha_{n}{ }^{2}\right) t}-1\right]} \\
\sigma_{r \phi}=\sum_{m, n, v=0}^{\infty}\left\{\left[a^{v}\left(1-v^{2}\right) r^{-v}-a^{-v}\left(v^{2}+v\right) r^{v}\right] \frac{J_{v-1}\left(\beta_{m} a\right)}{2 v \beta_{m} a}+\frac{v}{\beta_{m}{ }^{2} r^{2}}\left[\beta_{m} r J_{v-1}\left(\beta_{m} r\right)-(1+v) J_{v}\left(\beta_{m} r\right)\right]\right\} . \\
\lambda E \sin v\left(\phi-\phi^{\prime}\right) \sin \alpha_{n}\left(z+\frac{h}{2}\right) .\left[\bar{f}\left(\beta_{m}, v, \alpha_{n}\right)+\frac{\alpha}{2 \pi k} g_{s}{ }^{i} J_{v}\left(\beta_{m} r_{0}\right) \sin \alpha_{n}\left(\xi+\frac{h}{2}\right) e^{\alpha\left(\beta_{m}{ }^{2}+\alpha_{n}{ }^{2}\right) \tau}\right] \\
{\left[e^{-\alpha\left(\beta_{m}{ }^{2}+\alpha_{n}{ }^{2}\right) t}-1\right]}
\end{gathered}
$$

\section{Conclusion :}

In this paper we determined the temperature distribution and thermal stresses in a Dirichlet'sthin solid cylinder with moving heat source with analytical approach on the surface is established. By giving particular values to the parameter one can obtain their desired results by putting values of the parameters in the equations (5.10), (6.10), (6.11), (6.12)

\section{References}

[1] I. N. Sneddon The use of integral transform, McGraw Hill, New York, 1972

[2] M. NecatiOzisik `Heat conduction, Second Edition, A Wiley-Interscience Publication John Wiley and Sons, inc. New-York.

[3] N. Noda, R. B. Hetnarski, Y. Tanigawa, Thermal Stresses, second edition, 2002 